\title{
Automatic condition monitoring method to find defects in high- voltage insulators using infrared images
}

\author{
A. D. Zaripova ${ }^{1, *}$, D. K. Zaripov ${ }^{1}$, and A. E. Usachev ${ }^{1}$ \\ ${ }^{1}$ Kazan State Power Engineering University, Kazan, Russia
}

\begin{abstract}
In recent years, infrared imaging has become an important tool, particularly for predicting and preventing electrical equipment failure. Systems for online monitoring of the equipment conditions used in electrical substations are based on computer vision algorithms to perform visual analysis, automatically detect and assess equipment condition. This article describes a developed method that automatically finds defects in high-voltage insulators using infrared images. This method is based on the Otsu method, which is one of the most popular and effective segmentation methods that can be applied to finding defects in infrared images. The result is a comparative analysis of computer vision methods in infrared images used in our research. Automatic condition monitoring to find defects in high-voltage insulators in infrared images can be considered as the base method for an automated thermal imaging system for monitoring electrical substation equipment.
\end{abstract}

\section{Introduction}

Monitoring of electrical equipment or any of its components has become a challenging and important task. This is because of different equipment condition, which can result in various types of problems with the electrical equipment, but can be identified by detecting infrared radiation [1]. Degradation of electrical equipment and its components can cause overheating, which can lead to subsequent equipment failure, and can lead to unplanned power outages, possible injury and/or fire hazards. [2] Therefore, early prevention of equipment failure is required to avoid such situations, with the help of online condition monitoring systems at electrical substations.

Condition monitoring systems are based on continuous infrared image processing to assess the condition of electrical equipment [3].

The infrared image displays the temperature distribution on the object and the equipment defect is displayed as a "hot" (bright) spot. For the detection of "hot" spots in infrared images, computer vision methods are based on segmentation, clustering, searching for threshold values [4]. The purpose of these methods is to divide the image into its component parts to search for a region of interest to find equipment, background, faults and defects [5].

This article presents an automatic condition monitoring method for finding defects in high-voltage insulators in infrared images. This method is based on computer vision methods [6] for searching for a region of interest of images using Otsu segmentation method.

Typical elements of the power line and substation were chosen as initial objects for studying the capabilities of the method of automatically searching for defects in high-voltage insulators: a suspension polymer insulator for a voltage of $35 \mathrm{kV} \mathrm{LK70/35} \mathrm{and} \mathrm{a} \mathrm{porcelain}$ insulator for a voltage of $10 \mathrm{kV}$ IPU-10/630-7.5 UHL1 . In the high-voltage laboratory of KGEU, the insulators were supplied with a working voltage and thermograms (infrared images) were taken. Insulators are the most difficult to diagnose elements of high-voltage equipment [6].

The set of images (Figure 1) presents the following situations:

- two suspension insulators without defects;

- two bushing insulators on one of which (right) there is a transverse crack of a porcelain bus in the area of connection with the supporting flange;

- two contaminated suspension insulators after strong moisture;

- two contaminated suspension insulators after low moisture;

- two bushing insulators with poor contacts with a conductive bus;

- one suspension insulator without defects.

\section{Automatic condition monitoring method to find defects of a high-voltage insulators in infrared images}

The automatic condition monitoring method to find defects of high-voltage insulators in infrared images is implemented as a program in $\mathrm{C}++$ using the computer vision library OpenCV.

The automatic condition monitoring method is based on the following research results:

\footnotetext{
* Corresponding author: alina17@bk.ru
} 


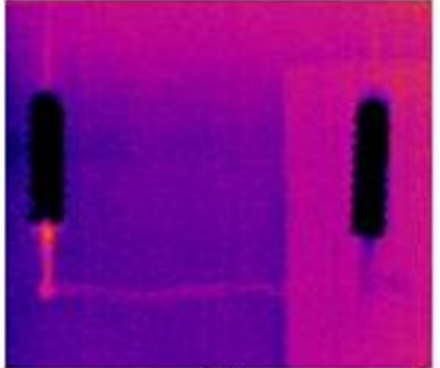

a)

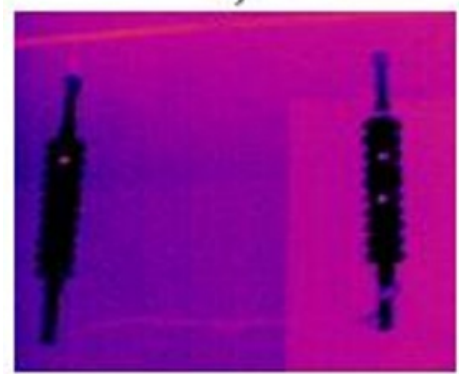

d)

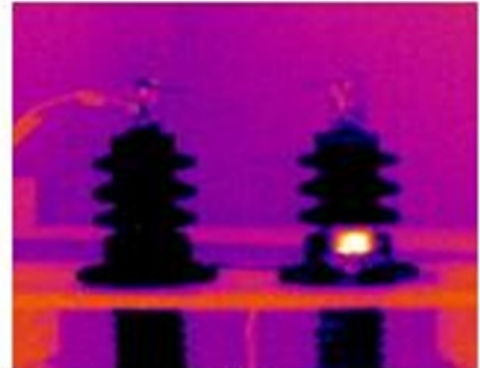

b)

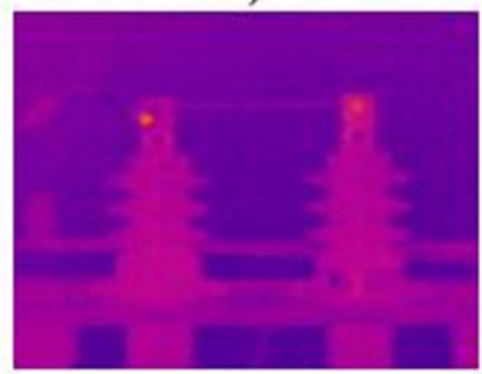

e)

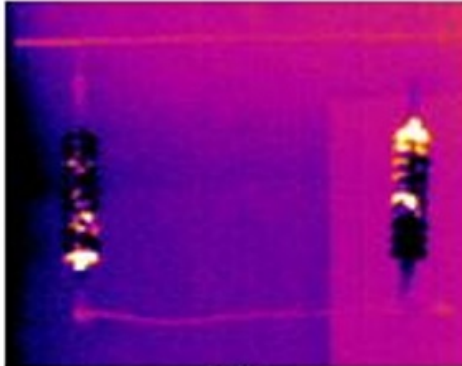

c)

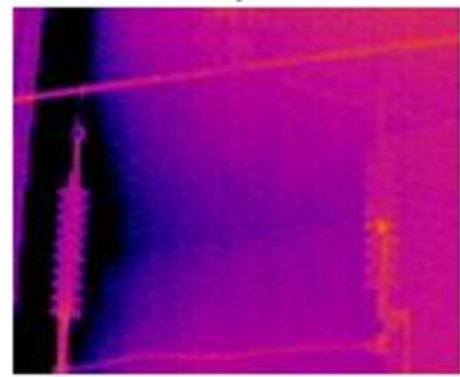

f)
Fig. 1. Infrared images of high-voltage insulators.

- criteria for equipment defects for thermal power substation control system based on the requirements of regulatory documents on thermal imaging control of electrical equipment and analysis of the extensive database of substation objects and high-voltage lines [7];

- comparative analysis of computer vision methods to search for defects in high-voltage insulators in infrared images. The result of this experiment demonstrated effectiveness of the Otsu method for identifying objects and qualitatively detecting the defects themselves [8].

The automatic condition monitoring method described in this article is based on the Otsu segmentation method, which is one of the simplest and most effective image segmentation methods. Image segmentation is one of the important tasks in image processing [9]. The purpose of segmentation is to divide the image into its constituent areas or objects [10]. The threshold processing technique has been widely used in various image segmentation tasks due to its simplicity and ease of implementation [11]. When studying the thermal state of electrical equipment, the threshold treatment method is a very useful tool for separating a warm region from its background [12]. Possible thermal anomalies in equipment can be detected by filtering the image using a certain threshold value [13].

The automatic condition monitoring method to find defects in high-voltage insulators is presented in the following diagram (Figure 2):

- Input image - infrared image of a high-voltage insulator is loaded into the program

- Convert image to grayscale - infrared image is converted to grayscale for subsequent segmentation using the Otsu method [13]

- Gaussian image filtering - infrared image is smoothed using a Gaussian filter to remove highfrequency noise
- Selecting an object using the Otsu method segmentation of the infrared image using the Otsu method

Defining hotspots using a threshold value determining the threshold value using the Otsu method and identifying hotspots in the infrared image.

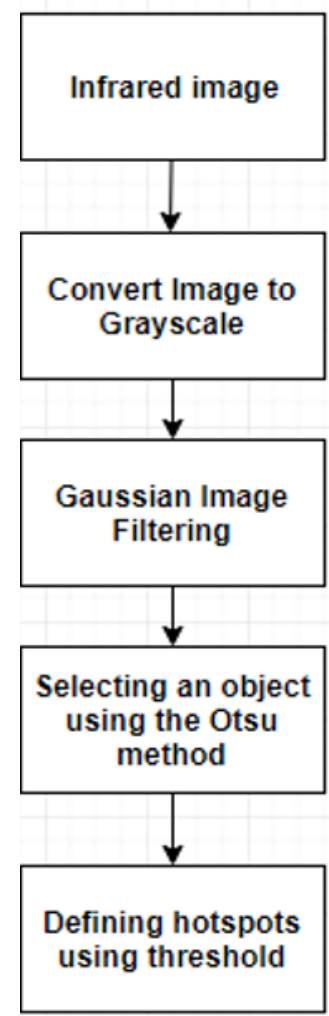

Fig. 2. Samples of infrared images of insulators, converted to grayscale. 


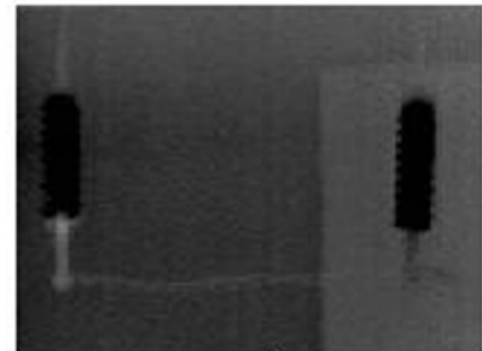

a)

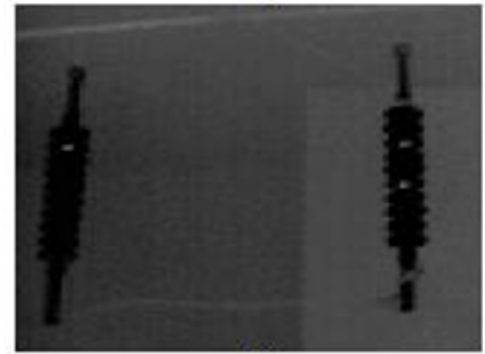

d)

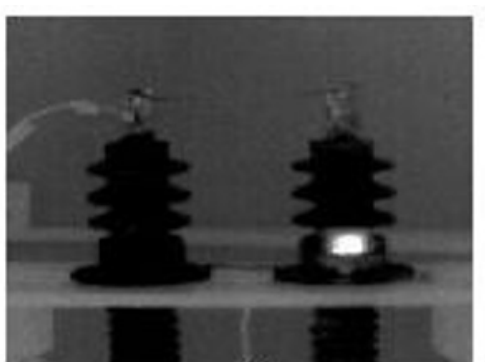

b)

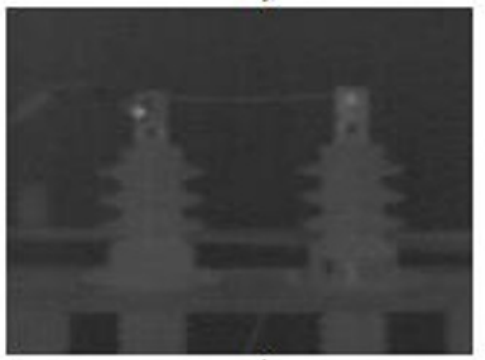

e)

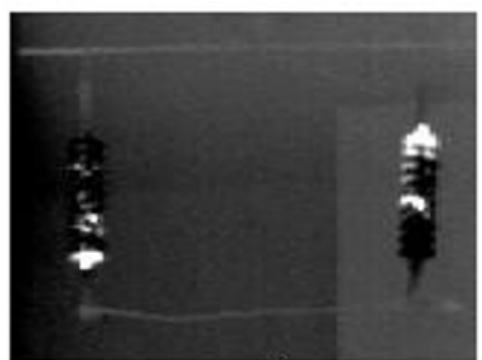

c)

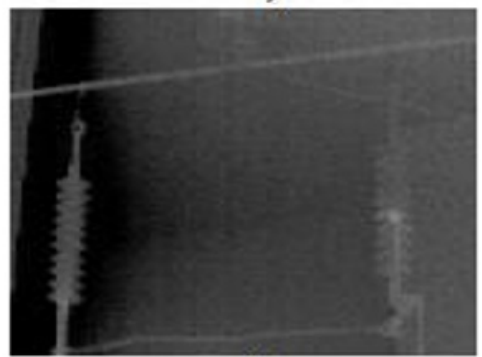

f)

Fig. 3. Samples of infrared images of insulators, converted to grayscale.

\section{Infrared image}

It is assumed that the electrical equipment and its components are hotter than their surroundings due to the effect of heat transfer.

A computer experiment was conducted on the following images (Figures 1, a-f):

- infrared image without defects;

- good quality infrared image with one defect;

- good quality infrared image with two defects;

- good quality infrared image with three defects;

- poor quality infrared image with two defects;

- infrared image without defects.

\section{Convert Image to Grayscale}

For the Otsu method, images need to be converted to grayscale [14].

\section{Gaussian Image Filtering}

Before image processing, infrared images are smoothed by Gaussian filter to remove high-frequency noise [15].

Then we apply Gaussian filter to images in grayscale.

A Gaussian filter is an image blur filter that uses normal distribution to calculate the transformation using formula (1) applied to each pixel of the image. The equation of the Gaussian distribution in $\mathrm{N}$ dimensions is:

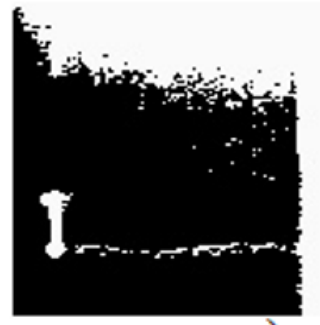

a)

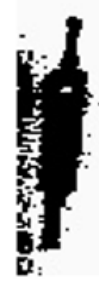

d)

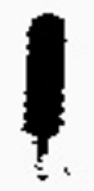

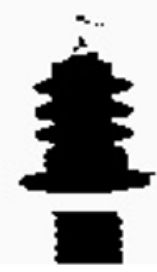

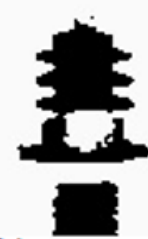

b)

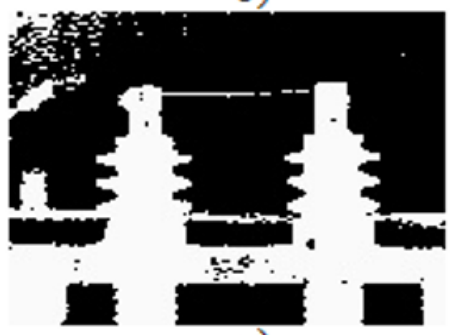

e)

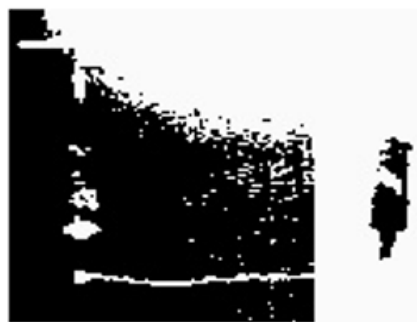

c)

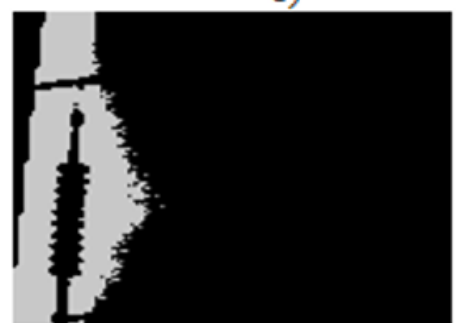

f)

Fig. 4. The result of processing infrared images of high-voltage insulators using the Otsu method. 


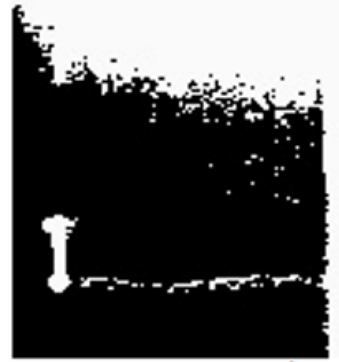

a)

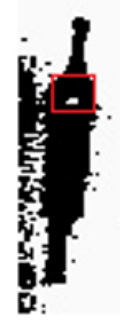

d)
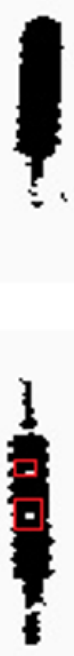

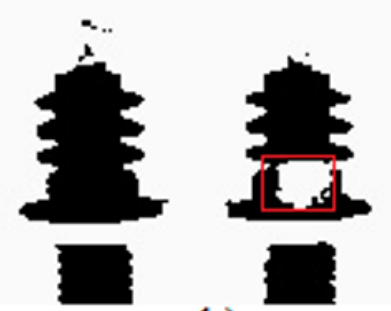

b)

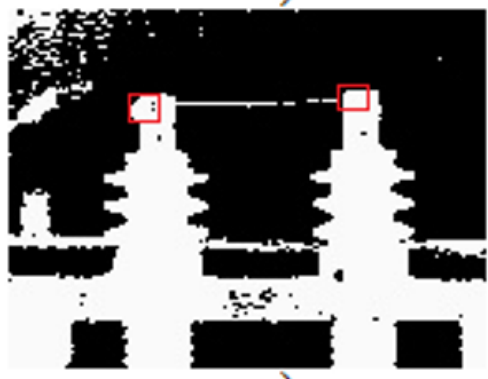

e)

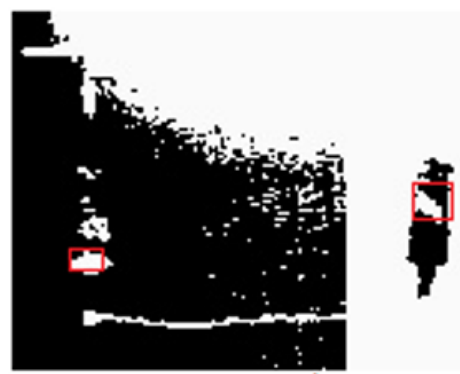

c)

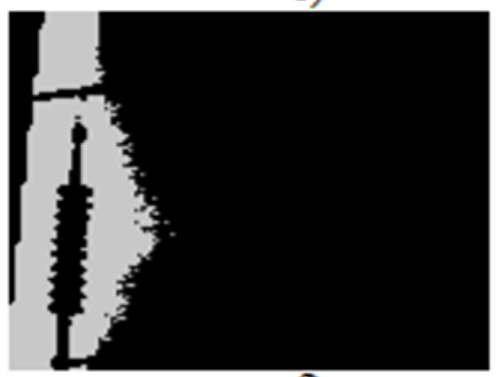

f)

Fig. 5. The result of processing infrared images of high-voltage insulators using the Otsu method.

$$
G(r)=\frac{1}{\left(2 \pi \sigma^{2}\right)^{\frac{N}{2}}} e^{\frac{-r^{2}}{\left(2 \sigma^{2}\right)}},
$$

where $\mathrm{r}$ is the blur radius, $r^{2}=u^{2}+v^{2}, \sigma$ is the standard deviation of the Gauss distribution.

\section{Selecting an object using the Otsu method}

The Otsu method is used to calculate the binarization threshold for a halftone image to separate an object from the background [13].

The Otsu method searches for a threshold using formula (2), which reduces variance within a class, which is defined as the weighted sum of variances of two classes:

$$
r_{\omega}^{2}=\omega_{1}(t) \sigma_{1}^{2}(t)+\omega_{2}(t) \sigma_{2}^{2}(t)
$$

where $\omega_{i}-$ is the probabilities of two classes divided by the threshold $\mathrm{t}, \sigma_{i}^{2}-$ is the variance of these classes.

The Otsu method shows that minimizing dispersion within a class is equivalent to maximizing dispersion between classes:

$$
\sigma_{b}^{2}=\sigma^{2}-\sigma_{\omega}^{2}(t)=\omega_{1}(t) \omega_{2}(t)\left[\mu_{1}(t)-\mu_{2}(t)\right]^{2},
$$

Formula (3) is expressed in terms of the probability $\omega_{i}$ and the arithmetic mean class $\mu_{i}$, which can be updated iteratively.

As a result of segmentation of infrared images of insulators using the Otsu method, the following (Figure 4) equipment objects were found on both highquality and poor-quality images.

\section{Defining hotspots using a threshold}

The Otsu method for each image determines the threshold value corresponding to the maximum dispersion $\sigma_{i}^{2}$. Defects were detected in infrared images

\begin{tabular}{|c|c|c|c|c|c|c|}
\hline & $\mathrm{a}$ & $\mathrm{b}$ & $\mathrm{c}$ & $\mathrm{d}$ & $\mathrm{e}$ & $\mathrm{f}$ \\
\hline Objects & 2 & 2 & 2 & 2 & 2 & 2 \\
\hline Defects & 0 & 1 & 2 & 3 & 2 & 1 \\
\hline Objects detected & 2 & 2 & 2 & 2 & 2 & 1 \\
\hline Defects detected & 0 & 1 & 2 & 3 & 2 & 0 \\
\hline Threshold & 58 & 38 & 55 & 40 & 125 & 85 \\
\hline $\begin{array}{l}\text { Hottest spot } \\
\text { coordinates }\end{array}$ & $104,119,0,2$ & $\begin{array}{l}127,119,0, \\
2\end{array}$ & $26,9,0,0,2$ & $\begin{array}{c}8,11,9,0, \\
2\end{array}$ & $151,119,0,2$ & $\begin{array}{c}25,1,19,0, \\
2\end{array}$ \\
\hline
\end{tabular}

Table 1. The result of processing infrared images using automatic condition monitoring method to find a defect on a highvoltage insulator. 
using threshold values (Figure 5). The table below shows the threshold values for each image and the coordinates of the hottest points in infrared images.

\section{Conclusions}

In this paper, we proposed automatic condition monitoring method for finding objects and defects in high-voltage insulators using infrared images. The correct region identification is very useful for evaluating the thermal condition of electrical equipment. However, finding objects and defects in infrared images is a very challenging task as the target regions always have irregular intensities, dim targets and very cluttered background. The experiment using proposed method has demonstrated that it is indeed a better defect detection technique compared to existing ones.

\section{References}

[1] R.A. Epperly, G.E. Heberlein, L.G. Eads, $A$ tool for reliability and safety: predict and prevent equipment failures with thermography (Petroleum and Chemical Industry Conference) 59-68 (1997)

[2] C. Hellier, Handbook of nondestructive evaluation. (McGraw-Hill Professional) 603 (2001)

[3] S. Taib, M. Sha, Infrared Image Enhancement and Segmentation for Extracting the Thermal Anomalies in Electrical, (Electronics and electrical engineering) 107-112 (2012)

[4] S. Taib, M. Sha, K.H. Ghazali, A.S.N Huda Finding ROIs in infrared image of electrical installation for qualitive thermal condition evaluation (IEEE International Conference on Control System, Computing and Engineering), 244-249 (2012)

[5] R. Gonzalez, Digital image processing. (Upper Saddle River N.J. Prentice Hall) 595-596. (2002)

[6] R. Szeliski, Computer Vision: Algorithms and Applications (2010)

[7] A.D. Zaripova, D.K. Zaripov, A.E. Usachev, Criteria for equipment defects for thermal power substation control system (Energy sector problems), 19(5-6), 50-56 (2017)

[8] A.D. Zaripova, D.K. Zaripov, A.E. Usachev, Visualization of high-voltage insulators defects in infrared images using computer vision methods (Scientific Visualization), 11(2), 88-98 (2019)

[9] M.S. Jadin, S. Taib, Infrared Image Enhancement and Segmentation for Extracting the Thermal Anomalies in Electrical Equipment, (Electronics and Electrical Engineering), 120(4), (2012)

[10] L. Ying, J.M. Xing, An efficient method for target extraction of infrared images // Artificial Intelligence and Computational Intelligence, (Springer Berlin Heidelberg) 6319, 185-192 (2010)

[11] M. Sezgin, B. Sankur, Survey over image thresholding techniques and quantitative performance evaluation (Journal of Electronic Imaging) 13(1), 146168 (2004)

[12] M.S. Jadin, S. Taib, K.H. Ghazali, Feature extraction and classification for detecting the thermal faults in electrical installations. (Measurement) 57, 15-24 (2014) 